\title{
The Effective Pressure of a Saturated Gluon Plasma
}

\author{
Adrian Dumitru and Miklos Gyulassy \\ Physics Department, Columbia University, 538W 120th Street, New York, NY 10027, USA
}

(November 13, 2018)

The evolution of the gluon plasma produced with saturation initial conditions is calculated via transport theory for nuclear collisions with $0.1<\sqrt{ } s<10 A \mathrm{TeV}$. The effective longitudinal pressure is found to remain significantly below the lattice QCD pressure with these initial conditions until the plasma cools to near the confinement scale. The absolute value of the transverse energy per unit of rapidity and its dependence on beam energy is shown to provide a sensitive test of gluon saturation models since the fractional transverse energy loss due to final state interactions is predicted to be much smaller and exhibit a weaker energy dependence than nondissipative hydrodynamics applied throughout the evolution.

There is an ongoing experimental program to produce a (transient) deconfined phase of QCD matter |1 in the laboratory via nuclear collisions at high energies [2]. The production mechanism is the liberation of a large number of gluons from the nuclear structure functions. The plasma is produced from copious minijet gluons at central rapidity, $y \simeq 0$, with transverse momentum $p_{T}>p_{0}$. The rapidity density of gluons liberated in central $A+A$ collisions can be estimated from [3]

$$
\begin{aligned}
\frac{\mathrm{d} N}{\mathrm{~d} y}\left(p_{0}\right)= & K T_{A A}(b=0) \int_{p_{T}>p_{0}} \mathrm{~d}^{2} p_{T} \int \mathrm{d} x_{a} \mathrm{~d} x_{b} \\
& G\left(x_{a}, p_{T}^{2}\right) G\left(x_{b}, p_{T}^{2}\right) \frac{\hat{s}}{\pi} \frac{\mathrm{d} \sigma}{\mathrm{d} \hat{t}} \delta(\hat{s}+\hat{t}+\hat{u})
\end{aligned}
$$

$\hat{s}, \hat{t}, \hat{u}$ are the Mandelstam variables for the partonparton scattering process, and $\mathrm{d} \sigma / \mathrm{d} \hat{t}$ denotes the hardscattering differential cross section in lowest order of perturbative QCD. $G\left(x, p_{T}^{2}\right)$ denotes the LO gluon distribution function in the nucleus. The phenomenological factor $K=2$ accounts approximately for NLO corrections. The nuclear overlap function $T_{A A}(0)=A^{2} / \pi R_{A}^{2}$ determines the number of binary nucleon collisions in head-on reactions within the Glauber approach, where $R_{A} \simeq 1.1 A^{1 / 3} \mathrm{fm}$ for mass $A$ nuclei.

For large $p_{0}$, the produced gluon plasma is dilute. As $p_{0}$ decreases, however, the density of gluons increases rapidly due to the increase of $G\left(x, p_{T}^{2}\right)$ as $x \approx 2 p_{T} / \sqrt{ } s$ decreases. It has been conjectured [4] that below some transverse momentum scale $p_{0} \leq p_{\text {sat }}$ the phase-space density of produced gluons may saturate since $g g \rightarrow g$ recombination could limit further growth of the structure functions. Phenomenologically, this condition may arise when gluons (per unit rapidity and transverse area $\pi / p_{\text {sat }}^{2}$ ) become closely packed and fill the available nuclear interaction transverse area. The saturation scale $p_{\text {sat }}$ can thus be estimated from

$$
\frac{\mathrm{d} N}{\mathrm{~d} y}\left(p_{\text {sat }}\right)=p_{\text {sat }}^{2} R_{A}^{2} / \beta,
$$

where $\beta \sim 1$. For $\beta=1$ the solution reported in EKRT 5 was

$$
\begin{aligned}
p_{\mathrm{sat}} & \approx 0.208 A^{0.128} \sqrt{s}^{0.191} \\
C_{1} & \approx 1.34 A^{-0.007} \sqrt{s}^{0.021},
\end{aligned}
$$

where $p_{\text {sat }}$ and $\sqrt{s}$ are in units of $\mathrm{GeV}$ and $C_{1}$ is the average transverse energy per gluon (in units of $p_{\text {sat }}$ ). The focus of this paper is to investigate whether the final observed $\mathrm{d} E_{T}^{f} / \mathrm{d} y$ can be used to test the predicted $A$ and $\sqrt{ } s$ dependence of the initial $\mathrm{d} E_{T}^{i} / \mathrm{d} y=$ $C_{1} p_{\text {sat }} \mathrm{d} N\left(p_{\text {sat }}\right) / \mathrm{d} y$.

Different gluon saturation models based on non-linear evolution and classical Yang-Mills equations [6,7] suggest that the factor $\beta$ in (2) may vary parametrically as

$$
\beta\left(p_{\text {sat }}\right)=\frac{4 \pi \alpha\left(p_{\text {sat }}\right) N_{c}}{c\left(N_{c}^{2}-1\right)},
$$

where $c \sim 1$ is a nonperturbative factor proportional to the fraction of the initial gluons in the nucleus which are liberated. This factor was recently estimated using lattice classical Yang-Mills methods [8] to be $c \approx 1.3$.

The first data [9] from $\mathrm{RHIC}$ on $\mathrm{Au}+\mathrm{Au}$ collisions at $\sqrt{ } s=130 A \mathrm{GeV}$ with $\mathrm{d} N_{c h} / \mathrm{d} \eta \approx 560$ is in fact reproduced by the EKRT saturation model [5] with $\beta=1$ assuming isentropic expansion (see [10]). On the other hand, a fit to the Phobos data using eq.(14) requires $c \approx 1.9$. Note that the solution of eq. (2) with $\beta \neq 1$ can be obtained from eq. (3) by rescaling the mass-number $A \rightarrow A / \beta^{2 / 3}$, and iterating until the stationary point is reached. Extrapolating to CERN-LHC energy the minijet multiplicities are predicted to be $\mathrm{d} N / \mathrm{d} y=3200$ for $\beta=1$ versus $\mathrm{d} N / \mathrm{d} y=5100$ for $\beta\left(p_{\text {sat }}\right)$ using eq. (4).

While $\mathrm{d} N(A, \sqrt{ } s) / \mathrm{d} y$ systematics provide one experimental handle to test different saturation and fixed scale models of initial conditions [10], another important observable that probes collective dynamics is the transverse energy per unit rapidity, $\mathrm{d} E_{T} / \mathrm{d} y$. In EKRT the final value of $\mathrm{d} E_{T}^{f} / \mathrm{d} y$ was predicted to be much smaller than produced initially due to collective longitudinal work assuming the validity of isentropic hydrodynamics. 
If the expansion proceeds in approximate local equilibrium with pressure $p=c^{2} \epsilon$ and speed of sound $c$, then the energy density, $\epsilon(\tau)$, must decrease faster than the expansion rate $\Gamma_{\text {exp }}=1 / \tau$ and leads to a bulk transverse energy loss

$$
\frac{E_{T}(\tau)}{E_{T}\left(\tau_{0}\right)}=\frac{\tau \epsilon}{\tau_{0} \epsilon_{0}}=\left(\frac{\tau_{0}}{\tau}\right)^{\delta}
$$

If local equilibrium is maintained during the evolution $\delta=c^{2}$. In contrast, if the system expands too rapidly to maintain local equilibrium, then the effective pressure is reduced (relative to that from LQCD) due to dissipation. The extreme asymptotically free plasma case corresponds to free streaming with $\delta=0 . E_{T}$ thus provides an important barometric observable that probes the (longitudinal) pressure in the plasma [11. There have been of course many studies on the magnitude of dissipative effects on this and other observables, see e.g. 12 16. The new twist on this old problem that we consider here is to extend those studies to the novel initial conditions suggested by gluon saturation models [5, 7, 8, .

To compute the transverse energy loss due to longitudinal work, we employ the Boltzmann equation in relaxation time approximation [12,16,17,

$$
p \cdot \partial f(p, x)=\Gamma_{r e l} p \cdot u\left(f_{e q}(p \cdot u)-f(p, x)\right) .
$$

$u^{\mu}$ denotes the four-velocity of the comoving frame and $f_{e q}$ is the chemical and thermal equilibrium phase space distribution, towards which $f$ evolves at a relaxation rate $\Gamma_{r e l}$. It is important to emphasize that this much simplified transport equation has been extensively tested against full $3+1 \mathrm{D}$ covariant parton cascade codes 14 and provides a surprisingly accurate equation for calculating the evolution of the transverse energy observable even in highly dissipative systems far from equilibrium $\left(\Gamma_{\text {rel }} \lesssim \Gamma_{\text {exp }}\right)$.

The relevant relaxation rate is given by the fractional energy loss per unit length,

$$
\Gamma_{r e l}=\frac{1}{E} \frac{\mathrm{d} E}{\mathrm{~d} z},
$$

which receives a contribution both from elastic and inelastic scattering,

$$
\begin{aligned}
\Gamma_{r e l} & =\rho \int\left(\mathrm{d} \sigma_{e l} \frac{\Delta E_{e l}}{E}+\mathrm{d} \sigma_{i n} \frac{\Delta E_{i n}}{E}\right) \\
& \approx \rho \int_{\mu^{2}}^{Q^{2}} \mathrm{~d} q^{2} \frac{\mathrm{d} \sigma_{e l}}{\mathrm{~d} q^{2}}\left\{\frac{q^{2}}{2 E^{2}}+\frac{\alpha N_{c}}{\pi} \int_{\mu^{2}}^{q^{2}} \frac{\mathrm{d} k_{T}^{2}}{k_{T}^{2}} \int \frac{\mathrm{d} x}{x} x\right\} \\
& =\rho\left(\frac{16 \pi \alpha^{2} N_{c}^{2}}{N_{c}^{2}-1}\right)\left(\frac{1}{s} \log \frac{Q^{2}}{\mu^{2}}+\frac{\alpha N_{c}}{\pi \mu^{2}} \log \frac{\overline{q^{2}}}{\mu^{2}}\right) .
\end{aligned}
$$

In these equations $\rho(\tau)$ denotes the gluon density in the local restframe, $\mu^{2}$ is the Debye screening mass in the medium, $Q^{2} \simeq s$ is the upper bound for the momentum transfer in the scattering process, and $x$ denotes the fraction of energy carried away by radiated gluons. In the last step we replaced the momentum transfer $q^{2}$ in the expression for the radiative energy loss by its average, $\overline{q^{2}} \approx \mu^{2} \log Q^{2} / \mu^{2}$. In local thermal equilibrium the average energy per gluon and the Debye screening scale are

$$
\begin{aligned}
\bar{s} / 2 & =(\epsilon / \rho)^{2} \simeq 9 T^{2} \\
\mu^{2} & =N_{c} g^{2} T^{2} / 3=4 \pi \alpha T^{2} .
\end{aligned}
$$

Assuming that the ratio $\bar{s} / \mu^{2}$ is essentially the same even out of equilibrium, the relaxation rate is approximately given by

$$
\Gamma_{r e l} \approx 9 \pi \alpha^{2} \frac{\rho^{3}}{\epsilon^{2}}\left(\log \frac{1}{\alpha}+\frac{27}{2 \pi^{2}}\right) \equiv K_{i n} 9 \pi \alpha^{2} \frac{\rho^{3}}{\epsilon^{2}} \log \frac{1}{\alpha} .
$$

We have set the double-logarithm of $Q^{2} / \mu^{2} \sim 1 / \alpha$ equal to unity. The expression (11) in fact overestimates the relaxation rate at early times, because the screening length $1 / \mu \sim 1 / g p_{\text {sat }}$ exceeds formally the horizon at $\tau_{0}=1 / p_{\text {sat }}$ for longitudinal Bjorken expansion [18 and because we neglect the suppression of radiation due to formation time physics. However, since with $p_{\text {sat }} \lesssim 2 \mathrm{GeV}, g \approx 2 \mathrm{up}$ to the LHC energy domain, we ignore this formal point in the discussion below.

The inelastic, radiative energy loss represents a significant source of uncertainty and is especially important in chemically undersaturated models of the initial conditions [15,19]. Within the saturation model, gluon multiplication through $2 \rightarrow 3$ processes may lead to thermalization of the soft radiated gluons at times parametrically large as compared to $\tau_{0}$, while the effect on the hard part of the gluon distribution is small [20]. In the present paper we do not attempt a more detailed treatment of radiative energy loss but simply vary the factor $K_{\text {in }} \sim 1-2$ to provide a measure of the theoretical uncertainites. We note that radiative energy loss for gluons with modest $p_{T}<5 \mathrm{GeV}$, is in any case significantly suppressed due to finite kinematic constraints and destructive interference effects [21].

The EKRT saturation model predicts the gluon density to be nearly chemically saturated already at the initial time $\tau_{0}=1 / p_{\text {sat }}$. This follows from the observation that ideal-gas formulas $\rho \sim T^{3}, \epsilon \sim T^{4}$ applied with chemical potential $\mu_{g}=0$ yield the same "temperature" $T_{0}$ [5].

At the initial time $\tau_{0}=1 / p_{\text {sat }}, \bar{s} / 2=\epsilon_{0}^{2} / \rho_{0}^{2}=C_{1}^{2} p_{\text {sat }}^{2}$. Therefore, noting that the comoving gluon density at time $\tau_{0}$ is $\rho_{0}=p_{\text {sat }}^{3} / \pi \beta$, the ratio of the relaxation rate to the expansion rate is given by

$$
\frac{\Gamma_{r e l}}{\Gamma_{\text {exp }}}=K_{i n} \frac{9 \alpha^{2}}{\beta C_{1}^{2}} \log \frac{1}{\alpha},
$$


While $\Gamma_{r e l} \propto p_{\text {sat }}$ increases as a power of the energy in eq. (3), the Bjorken boundary conditions 18 force the system to expand londitudinally initially also at an increasing rate $\Gamma_{\exp }\left(\tau_{0}\right)=p_{\text {sat }}$. The essential quantity that fixes the magnitude of the effective pressure relative to that predicted by LQCD is the ratio of rates in Eq. (12), which dimensionally is simply a function of $\alpha\left(p_{\text {sat }}\right)$. The asymptotic freedom property [22] of QCD therefore requires that this ratio vanishes as $\sqrt{ } s \rightarrow \infty$. In (12) the rate of how fast it vanishes is controlled by $\alpha^{2} K_{\text {in }}\left(p_{\text {sat }}\right) / \beta\left(p_{\text {sat }}\right)$. Therefore, with saturation initial conditions, asymptotic freedom reduces the effective pressure acting at early times $\tau \sim \tau_{0}$ and causes the initial evolution to deviate from ideal hydrodynamics for a time interval that, as we show below, increases with energy.

The total number of interactions during the evolution up to time $\tau$ is given by

$\phi(\tau) \equiv \int_{\tau_{0}}^{\tau} \mathrm{d} \tau^{\prime} \Gamma_{r e l}\left(\tau^{\prime}\right) \simeq \frac{K_{i n}}{\beta} \frac{9 \alpha^{2} \log (1 / \alpha)}{C_{1}^{2}} \log \frac{\tau}{\tau_{0}}$.

The last expression holds close to the free streaming regime ("Knudsen limit"), where the number of scatterings increases only logarithmically with time. We find that $\phi$ reaches on the order of unity at $\tau^{*} \approx 0.4 \mathrm{fm}$ in the BNL-RHIC to CERN-LHC energy region. However, the local relaxation rate at $\tau^{*}$ is still less than or on the order of the expansion rate $\left(1 / \tau^{*}\right)$. In a non-expanding plasma $\phi \sim 1$ provides a rough equilibration criterion. However, as long as $\Gamma_{r e l}$ is not significantly larger than $\Gamma_{\text {exp }}$ this criterion is insufficient to address how much collective hydrodynamic work can be done by the plasma.

For a quantitative estimate, we must solve the kinetic equations (6). The first energy moment of that equation together with energy conservation to replace $\epsilon_{e q}$ by $\epsilon(\tau)$, results in the energy density evolution equation 12, 16,17

$$
e^{\phi} \frac{\tau \epsilon}{\tau_{0} \epsilon_{0}}=1+\int_{0}^{\phi} \mathrm{d} \phi^{\prime} e^{\phi^{\prime}} \frac{\tau^{\prime}\left(\phi^{\prime}\right) \epsilon\left(\phi^{\prime}\right)}{\tau_{0} \epsilon_{0}} h\left(\frac{\tau^{\prime}\left(\phi^{\prime}\right)}{\tau(\phi)}\right) .
$$

This equation applies if the initially produced partons have a vanishing longitudinal momentum spread in the comoving frame, i.e. assuming a strong correlation between space-time rapidity and momentum space rapidity [18]. The function $h(x)$ appearing in (14) is given by $2 h(x) \sqrt{1-x^{2}}=x \sqrt{1-x^{2}}+\arcsin \sqrt{1-x^{2}}$; it insures that $\delta \rightarrow 1 / 3$ as $\tau \rightarrow \infty$.

Expanding (14) to first order in $\phi$ yields

$$
\frac{\tau \epsilon}{\tau_{0} \epsilon_{0}}=1-\left(\frac{3}{4}-\frac{\pi^{2}}{16}\right) \phi+\mathcal{O}\left(\phi^{2}\right)
$$

giving for $\tau \sim \tau_{0}$

$$
\delta=\left(\frac{3}{4}-\frac{\pi^{2}}{16}\right) \frac{9 K_{i n} \alpha^{2}}{\beta C_{1}^{2}} \log \frac{1}{\alpha} .
$$

Note that $\delta \rightarrow 0$ as $p_{\text {sat }} \rightarrow \infty$ in accordance with the discussion above.

For our numerical estimates we let $\alpha$ creep with time. Since the effective temperature scales as $\epsilon(\tau)^{1 / 4}$, the effective coupling, $\alpha\left(T_{\text {eff }}\right)$, increases slowly with time approximately as

$$
\alpha(\tau)=\left(\frac{12 \pi}{27}\right) / \log \left(1+\frac{p_{\mathrm{sat}}^{2}}{\Lambda_{Q C D}^{2}}\left(\frac{\epsilon(\tau)}{\epsilon\left(\tau_{0}\right)}\right)^{1 / 2}\right),
$$

with $\Lambda_{Q C D}=200 \mathrm{MeV}$. Furthermore, longitudinal expansion constrains $\rho(\tau) \tau$ to remain constant. As the system cools, the mean center of mass energy in collisions also decreases as

$$
\bar{s}(\tau)=2\left(\frac{\epsilon(\tau)}{\rho(\tau)}\right)^{2}=\bar{s}\left(\tau_{0}\right)\left(\frac{\tau \epsilon(\tau)}{\tau_{0} \epsilon\left(\tau_{0}\right)}\right)^{2} .
$$

The number of collisions between $\tau_{0}$ and $\tau$ in this case is given by

$$
\begin{aligned}
\phi(\tau)= & \Gamma_{r e l}\left(\tau_{0}\right) \int_{\tau_{0}}^{\tau} d \tau^{\prime}\left(\frac{\alpha\left(\tau^{\prime}\right)}{\alpha\left(\tau_{0}\right)}\right)^{2}\left(\frac{\epsilon\left(\tau_{0}\right)}{\epsilon\left(\tau^{\prime}\right)}\right)^{2}\left(\frac{\tau_{0}}{\tau^{\prime}}\right)^{3} \\
& \times \frac{\log \left(1+1 / \alpha\left(\tau^{\prime}\right)\right)}{\log \left(1+1 / \alpha\left(\tau_{0}\right)\right)} .
\end{aligned}
$$

We regulated the logarithmic dependence above for numerical stability. Note that with eq. (19), eq. (14) is a nonlinear self-consistency equation for $\epsilon(\tau)$.

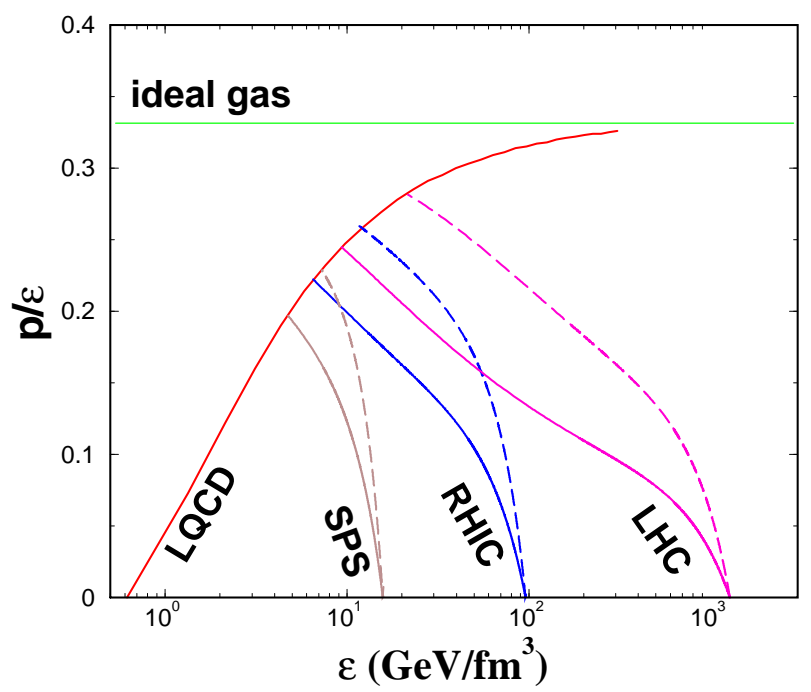

FIG. 1. The ratio of effective longitudinal pressure to energy density as a function of the energy density along the dynamical path is shown for SPS, RHIC, and LHC saturation initial conditions [5. Solid (dashed) curves are for $\beta=1$ and $K_{\text {in }}=1(2)$. The LQCD equation of state [1] is also shown for comparison.

In Figure 1 we show the effective longitudinal pressure as a function of the energy density for $\sqrt{ } s=20,200,5400$ 
A $\mathrm{GeV}$ saturation initial conditions. The ratio $p / \epsilon$ is defined here by $\delta(\tau)$ as obtained solving eq. (14) numerically. Our definition of the effective pressure absorbes all disspative corrections to the perfect fluid equation, $u_{\mu} \partial_{\nu}\left[(\epsilon+p) u^{\mu} u^{\nu}-p g^{\mu \nu}\right]=\mathrm{d} \epsilon / \mathrm{d} \tau+(\epsilon+p) / \tau=0$. For comparison the pressure of equilibrium QCD is also shown for $N_{f}=3$. This curve is obtained from the $N_{f}=0$ lattice data of [1] rescaling the number of relativistic degrees of freedom by $47.5 / 16$, and assuming a transition temperature $T_{c}\left(N_{f}=3\right)=160 \mathrm{MeV}$.

Initially $p / \epsilon$ starts at zero in this model and remains small for a large time relative to $1 / p_{\text {sat }}$ because the plasma is torn apart by the initial rapid longitudinal expansion. The effective pressure approaches the LQCD curve from below and reaches it at a time $\tau_{L} \approx 1-2 \mathrm{fm}$ at RHIC $\sqrt{ } s=200 \mathrm{AGeV}$, by which time the energy density has dropped by an order of magnitude, $\epsilon_{L} \equiv \epsilon\left(\tau_{L}\right)=$ $6.5-12 \mathrm{GeV} / \mathrm{fm}^{3}$. For LHC $\sqrt{ } s=5400 \mathrm{AGeV}, \tau_{L}=$ $3-7 \mathrm{fm}$ during which the energy density falls by almost two orders of magnitude to $\epsilon_{L}=9.5-21.5 \mathrm{GeV} / \mathrm{fm}^{3}$. The quoted intervals correspond to $K_{\text {in }}=1-2$ using the EKRT parametrization (3). The contrast between the dynamical path followed by the saturated plasma compared to the equilibrium equation of state is striking. A qualitatively similar behavior of the early logitudinal pressure has also been found from solutions of diffusion equations 23].

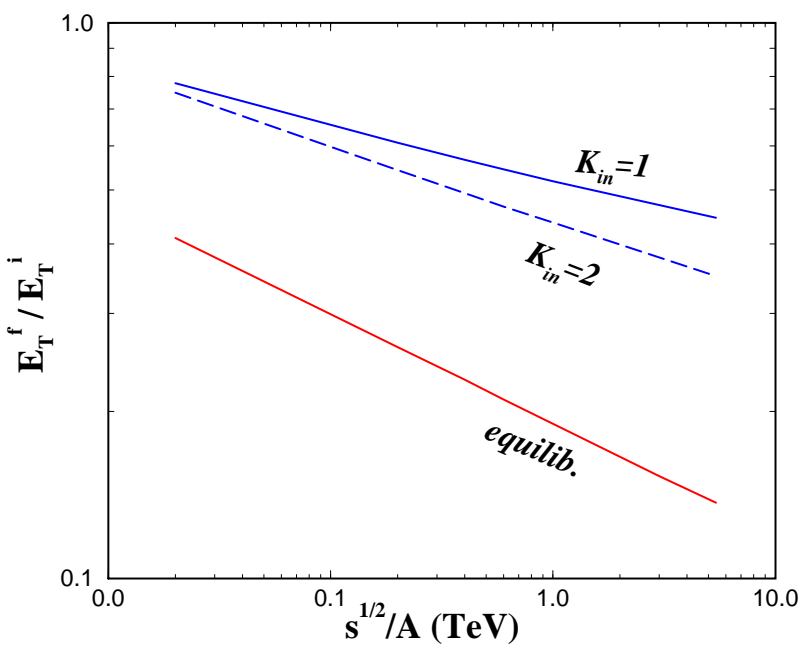

FIG. 2. The ratios of the final to the initial transverse energy per unit rapidity are shown as a function of beam energy for central $\mathrm{Au}+\mathrm{Au}$ collisions. The initial value corresponds to the EKRT parametrization $(\beta=1)$ and the transport results are for $K_{i n}=1,2$. For comparison, the final transverse energy assuming that local equilibrium was maintained throughout the evolution is also shown.

Since the longitudinal gradients at $\tau_{L}$ are much smaller than at $\tau_{0}$, the evolution beyond $\tau_{L}$ is much more likely to follow isentropic hydrodynamics along the lattice QCD equation of state. In this case one could calculate detailed differential hadronic observables along the same lines as in 24] using the conditions at $\tau_{L}$ as the initial conditions for $3+1 \mathrm{D}$ hydrodynamics.

The main experimentally observable consequence of the reduced effective pressure is shown in Fig. 2. The ratio $E_{T}^{f} / E_{T}^{i}=\tau_{f} \epsilon_{f} / \tau_{0} \epsilon_{0}$ has been obtained from the solution of the transport equation assuming $\epsilon_{f}=2 \mathrm{GeV} / \mathrm{fm}^{3}$ which corresponds roughly to $T \simeq T_{c}$. $\tau_{f}$ is estimated assuming hydrodynamic expansion from the point were the trajectories in Fig. 11 reach the LQCD curve at time $\tau_{L}$, with the equation of state $p / \epsilon=a+b \log \epsilon(\epsilon$ in units of $\left.\mathrm{GeV} / \mathrm{fm}^{3}\right)$. The parameters $a=0.051, b=0.092$ provide a reasonable fit to the LQCD curve shown in Fig. 1. In this case,

$$
\frac{\tau_{f}}{\tau_{L}}=\left(\frac{1+a+b \log \epsilon_{L}}{1+a+b \log \epsilon_{f}}\right)^{1 / b} .
$$

On the other hand, if ideal hydrodynamics were applicable already at $\tau_{0}$, the final observed transverse energy for $1+1$ dimensional adiabatic expansion would be

$$
\frac{E_{T}^{f}}{E_{T}^{i}}=\frac{\tau_{f} \epsilon_{f}}{\tau_{0} \epsilon_{0}}=\frac{\tau_{f}\left(T_{f} s_{f}-p_{f}\right)}{\tau_{0}\left(T_{0} s_{0}-p_{0}\right)}=\frac{T_{f}}{T_{0}} .
$$

The last step follows both for $p_{0, f} \simeq 0$ as well as $p_{0, f}=T_{0, f} s_{0, f} / 4$ from the condition of entropy conservation, $\tau s=$ const $[5]$. Strong transverse expansion leads to slightly larger $E_{T}^{f}$ but we shall neglect that small effect here for simplicity. Clearly, for $T_{f} \sim T_{c} \approx 160 \mathrm{MeV}$ one would observe a much smaller transverse energy in the final state than in the initial state. Moreover, $E_{T}^{f} / E_{T}^{i}$ would also have significantly stronger energy dependence such that $E_{T}^{f}$ deviates more and more from $E_{T}^{i}$ with increasing $\sqrt{ } s$. In this sense isentropic hydrodynamics erases information on the interesting initial conditions via this observable. The solutions of the transport equations clearly show a smaller decrease of $E_{T}^{f}$ and of the logarithmic slope, $\kappa=\mathrm{d} \log E_{T}^{f} / \mathrm{d} \log \sqrt{ } s$, due to final state interactions. We find that $\kappa=0.50$ for the initial state (3) evolved with $K_{\text {in }}=1, \kappa=0.46$ with $K_{\text {in }}=2$, while $\kappa=0.40$ with isentropic expansion, eq. (21). For comparison, the initial EKRT saturated $E_{T}^{i}=\pi R_{A}^{2} \tau_{0} \epsilon_{0}$ scales with the higher power $\kappa=0.59$ according to eq. (3). The fractional transverse energy loss is thus less dependent on energy than for entropy conserving expansion for which $E_{T}^{f} / E_{T}^{i} \propto 1 / T_{0} \propto 1 / \sqrt{s}^{0.2}$. This is due to the increasingly long time spent far from equilibrium in Fig. 1 as the beam energy increases.

The results in Fig. 2 are encouraging from the point of view of searching for evidence of gluon saturation in nuclei at high energies. Experimental data on $\mathrm{d} E_{T} / \mathrm{d} y$ or $\mathrm{d} E_{T} / \mathrm{d} \eta$ for central $\mathrm{Au}+\mathrm{Au}$ collisions at RHIC will soon provide a new test of saturation and non-saturation models at those energies. Since we predict that dissipative effects reduce considerably the effective longitudinal 
pressure in Fig. 1, the beam energy dependence of the transverse energy is expected to reflect much more accurately the predicted power law dependence of the initial conditions as seen in Fig. 2. We therefore conclude that the energy and $A$ systematics of the bulk calorimetric observable, $\mathrm{d} E_{T} / \mathrm{d} y$, will be a sensitive test of saturation models of gluon plasmas produced in the RHIC to LHC energy range.

\section{ACKNOWLEDGMENTS}

We thank K. Eskola, K. Kajantie, L. McLerran, A.H. Mueller, D. Son, R. Venugopalan and K. Tuominen for helpful criticism and discussions on thermalization aspects and saturation. We thank the BNL nuclear theory group for hosting a stimulating workshop during which this work was completed. We acknowledge support from the DOE Research Grant, Contract No.DE-FG-02-93ER40764 .

[1] G. Boyd, J. Engels, F. Karsch, E. Laermann, C. Legeland, M. Lutgemeier and B. Petersson, Nucl. Phys. B469 (1996) 419; M. Okamoto et al. [CP-PACS Collaboration], Phys. Rev. D60 (1999) 094510.

[2] Proc. 14th Int. Conf. on Ultrarelativistic NucleusNucleus Collisions (Quark Matter 99), Torino, Italy, 10-15 May 1999, Edited by L. Riccati, M. Masera, E. Vercellin, Nucl. Phys. A661 (1999) 1c, and references therein.

[3] J.C. Collins, D.E. Soper and G. Sterman, Nucl. Phys. B261 (1985) 104; K. Kajantie, P.V. Landshoff and J. Lindfors, Phys. Rev. Lett. 59 (1987) 2527; X. Wang and M. Gyulassy, Phys. Rev. D44 (1991) 3501.

[4] L.V. Gribov, E.M. Levin and M.G. Ryskin, Phys. Rept. 100 (1983) 1; A.H. Mueller and J. Qiu, Nucl. Phys. B268 (1986) 427.

[5] K.J. Eskola, K. Kajantie, P.V. Ruuskanen and K. Tuominen, Nucl. Phys. B570 (2000) 379.

[6] L. McLerran and R. Venugopalan, Phys. Rev. D49 (1994) 2233 and 3352;

[7] J.P. Blaizot and A.H. Mueller, Nucl. Phys. B289 (1987) 847; A.H. Mueller, Nucl. Phys. B572 (2000) 227.

[8] A. Krasnitz and R. Venugopalan, Phys. Rev. Lett. 84 (2000) 4309; and hep-ph/0004116.

[9] B.B. Back et al., PHOBOS Collaboration, hepex/0007036;

[10] X. Wang and M. Gyulassy, nucl-th/0008014.

[11] M. Gyulassy and T. Matsui, Phys. Rev. D29 (1984) 419.

[12] A. Hosoya and K. Kajantie, Nucl. Phys. B250 (1985) 666 ;

[13] P. Danielewicz and M. Gyulassy, Phys. Rev. D31 (1985) 53.
[14] M. Gyulassy, Y. Pang and B. Zhang, Nucl. Phys. A626 (1997) 999; D. Molnar and M. Gyulassy, nuclth/0005051

[15] S.M. Wong, Phys. Rev. C54 (1996) 2588; Phys. Rev. C56 (1997) 1075.

[16] H. Heiselberg and X. Wang, Phys. Rev. C53 (1996) 1892.

[17] G. Baym, Phys. Lett. B 138 (1984) 18; K. Kajantie and T. Matsui, Phys. Lett. B 164 (1985) 373; G. Baym, H. Monien, C.J. Pethick and D.G. Ravenhall, Nucl. Phys. A525 (1991) 415c.

[18] J.D. Bjorken, Phys. Rev. D27 (1983) 140.

[19] K.J. Eskola, K. Kajantie and J. Lindfors, Phys. Lett. B214 (1988) 613; K. Geiger and J.I. Kapusta, Phys. Rev. D47 (1993) 4905; T.S. Biro, E. van Doorn, B. Müller, M.H. Thoma and X.N. Wang, Phys. Rev. C48 (1993) 1275; L. Xiong and E. Shuryak, Phys. Rev. C49 (1994) 2203.

[20] R. Baier, A.H. Mueller, D. Schiff and D. Son, manuscript in preparation.

[21] M. Gyulassy, P. Levai, I. Vitev, nucl-th/0005032; nuclth/0006010.

[22] D.J. Gross and F. Wilczek, Phys. Rev. Lett. 30 (1973) 1343; H.D. Politzer, Phys. Rev. Lett. 30 (1973) 1346.

[23] J. Bjoraker and R. Venugopalan, hep-ph/0008294.

[24] A. Dumitru and D.H. Rischke, Phys. Rev. C59 (1999) 354. S.A. Bass and A. Dumitru, Phys. Rev. C61 (2000) 064909. 